\title{
Research and Parameter Optimization of the Infrared Sensor for Eye Track
}

\author{
M. ŻYCzkowski ${ }^{a, *}$, M. Szustakowski ${ }^{a}$, K. RóżAnOwski $^{b}$, K. Murawski $^{c}$, \\ M. KAROL ${ }^{a}$ AND P. MARKOWSKI ${ }^{a}$ \\ ${ }^{a}$ Institute of Optoelectronics, Military University of Technology \\ S. Kaliskiego 2, 00-908 Warsaw, Poland \\ ${ }^{b}$ Aviation Bioengineering Department, Military Institute of Aviation Medicine \\ Z. Krasińskiego 54, 01-755 Warsaw, Poland \\ ${ }^{c}$ Institute of Teleinformatics and Automatics, Military University of Technology \\ S. Kaliskiego 2, 00-908 Warsaw, Poland
}

\begin{abstract}
This paper presents infrared sensor for eye tracking system for ophthalmology and truck drivers. System is based on camera and near infrared diode illuminator. We present test results of sensor detection at different operating parameters. Tests were chosen so as to see how the sensor behaves in difficult conditions, additionally tested whether long-term operation of the sensor has an impact on the examined person.
\end{abstract}

PACS: 42.30.Wb, 87.57.-s, 42.79.Qx

\section{Introduction}

The subject of research is an optical sensor designed for subsystem of eye activity research (PBAO) [1, 2]. Sensor is used in two configurations. The first is used to study the changes of the eyes of people using contact lenses, the second is used to assess the state of the driver's mental and physical activity based on the recorded eye activity (Fig. 1). To test eye condition, distance from the illuminator to the eye is about $36 \mathrm{~cm}$, and in the test of driver's condition the distance depends on the seat position and it is about $80-110 \mathrm{~cm}, 25 \mathrm{~cm}$ below the eye level and is bent upwards at an angle of $15^{\circ}$.
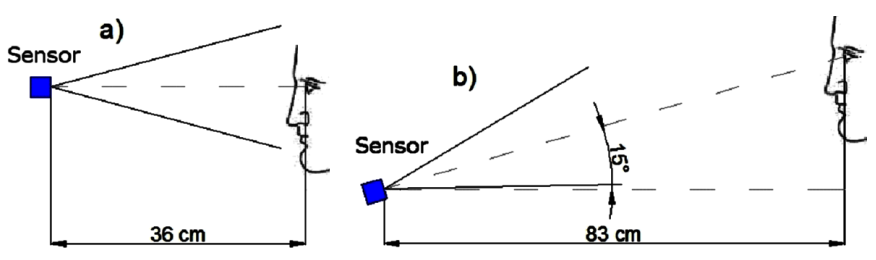

Fig. 1. Configuration to test eye condition (a) and tracking driver's eye (b).

The sensor is made up of monochrome camera, illumination controller, lens and filter. Illuminator is made of 12 LEDs designed to ensure optimum image capture to

\footnotetext{
* corresponding author; e-mail: mzyczkowski@wat.edu.pl
}

allow the use of dedicated image processing algorithms. The main purpose of the study was to determine the safety of the illumination, used in sensor, for eyesight. In addition, tests were conducted to answer the possibility of optimizing the parameters of illumination for better lighting conditions, and thus improve the performance of target systems. Optimization had to reveal the scope of the spectral power distribution of LEDs and the modulation frequency possible to make effective use of application systems. It was important from the perspective of improving the operation of software algorithms used for detection of the activity of the eye. Not in all cases, the maximum acceptable radiation power for the eye allowed the correct exposure because there were artifacts, reflections, or saturation.

\section{Measurements}

Because dedicated terminal devices using illuminator have been applied as paramedical and expose user to look directly at LED matrix of illuminator, measurements were made for comparison with current national and international guidelines for the safe work with the optical range of electromagnetic radiation.

Measurement station consists of:

a) spectrum analyzer (Yokogawa AQ 6370C) is used to determine the spectral characteristics of illuminator,

b) power meter (Thorlabs PM100D) S121C-probe controlled by a computer is used to determine the 
parameters of the radiation power of the illuminator.

Used in illuminator LED HIRB5-43G-A according to the manufacturer should emit radiation at a wavelength of $850 \mathrm{~nm}$. (The measured radiation patterns are shown at Fig. 2.) Also shows that the radiation power decreases with time and approaches the maximum wavelength of radiation by the manufacturer.

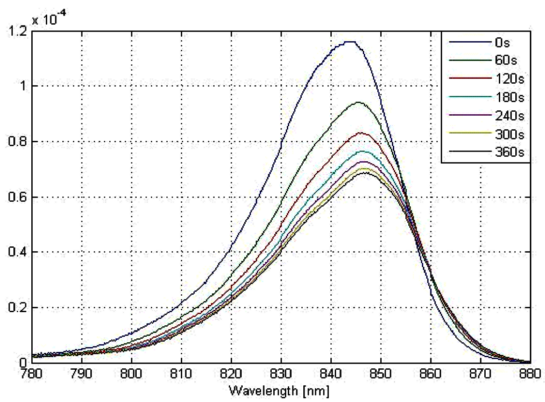

Fig. 2. Spectral characteristic of LED used in illuminator.

Because of the long term exposure to radiation of the driver eye, illuminator has necessary was to test stability of the generated radiation over time. Measurements were performed at a distance of $36 \mathrm{~cm}$ from the illuminator. Long-term tests have shown that the radiation power is greatest immediately after starting the device, then decreases to a constant level in about 10 min. After this time the changes to radiation power are minimal as shown in Fig. 3.

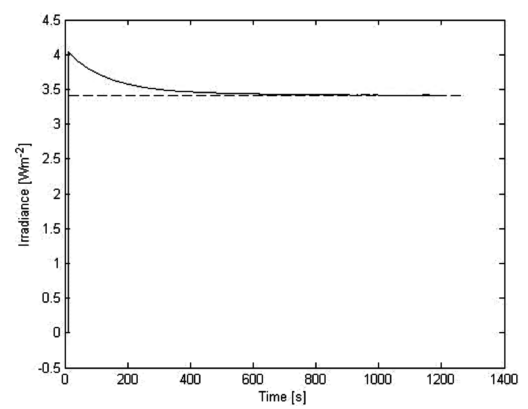

Fig. 3. Stability of illuminator radiation.

To determine the safety of the device it was necessary to investigate the radiation power depending on the distance between the eye and the illuminator (Fig. 4). Measurements were carried out at maximum power illuminator at a distance of $36-106 \mathrm{~cm}$ in steps of $2 \mathrm{~cm}$.

In addition, cross-beam illuminator was examined to determine the surface area illuminated by the illuminator and the possible effects of radiation generated in the other persons (Fig. 5). The measurements were conducted within $\pm 45^{\circ}$ from the optical axis of the camera at a distance $36 \mathrm{~cm}$.

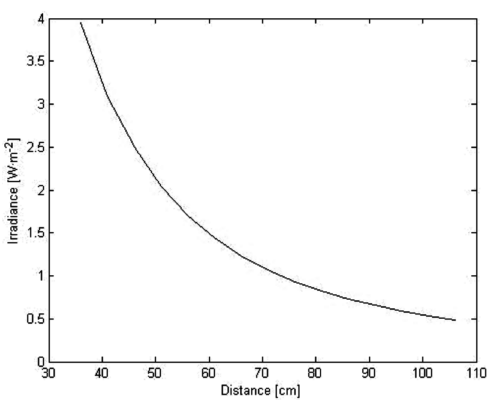

Fig. 4. Irradiance as a function of distance between eye and illuminator.

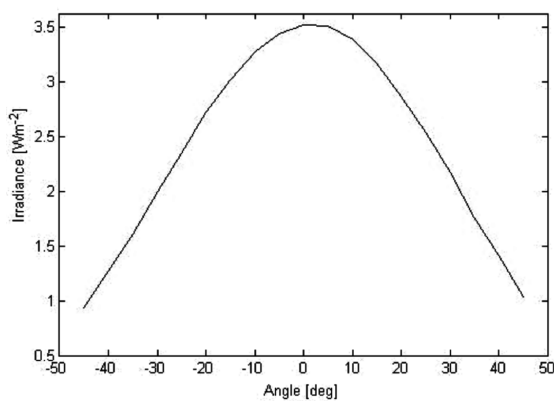

Fig. 5. Irradiance profile of illuminator.

The radiation intensity illuminations at the illuminated surface were also examined. These measurements allowed determining the homogeneity of illumination of the test area. Measurements were performed in the area $10 \times 10 \mathrm{~cm}^{2}$ with $0.5 \mathrm{~cm}$ step between measurements for the first configuration where camera field of view is of size approximately $6 \times 4 \mathrm{~cm}^{2}$, and in an area of $20 \times 20 \mathrm{~cm}^{2}$ $1 \mathrm{~cm}$ step between measurements for the second configuration where camera field of view is of size approximately $15 \times 11 \mathrm{~cm}^{2}$.

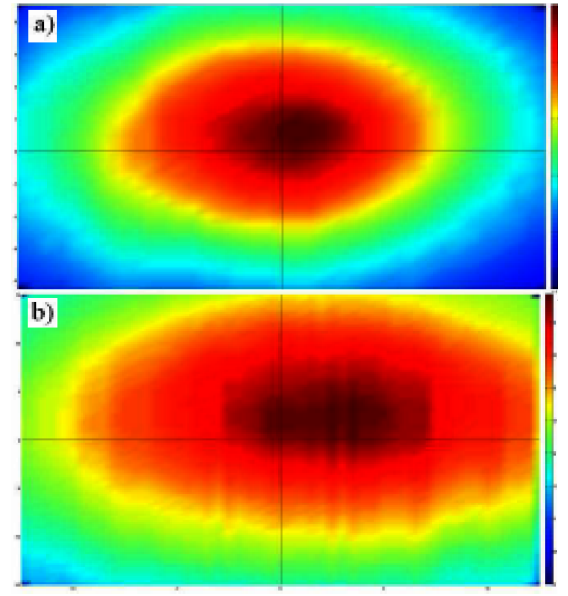

Fig. 6. The distribution of radiation power of illuminator at configuration 1 , area $12.5 \times 12.5 \mathrm{~cm}^{2}$ (a) and configuration 2 , area $25 \times 25 \mathrm{~cm}(\mathrm{~b})$. 
In Fig. 6 there are presented spectral power distribution graphs of illuminator in two configurations. Measurements were performed after setting the LED for optimum parameters for image by the camera. You can see that the distribution of radiation in the area observed by the camera is fairly uniform, so the risk of errors associated with image analysis of non-uniform lighting is minimal.

\section{Exposure limits and hazard evaluation}

To ensure the required safety of persons using the sensor has to be fulfilled by all of its components of safety standards. The current Polish standards of safety in regard to devices with emitters are determined in [3] and [4].

On the basis of these standards there have been designated maximum permissible exposures (MDE) to radiation illuminator (845 $\mathrm{nm}$ ) according to Eqs. (1) and (2), respectively, for the standards $[3,4]$ :

$$
\begin{aligned}
& \mathrm{MDE}=18 C_{4} C_{6} C_{7} T_{2}^{-0.25} \frac{\mathrm{W}}{\mathrm{m}^{2}}=11.10 \frac{\mathrm{W}}{\mathrm{m}^{2}}, \\
& \mathrm{MDE}=3.2 C_{4} \frac{\mathrm{W}}{\mathrm{m}^{2}}=6.24 \frac{\mathrm{W}}{\mathrm{m}^{2}},
\end{aligned}
$$

where $C_{4}=10^{\frac{\lambda-700 \mathrm{~nm}}{500 \mathrm{~nm}}}=1.95, C_{6}=C_{7}=1, T_{2}=100 \mathrm{~s}$.

The maximum radiation power measured during the test illuminator on a minimum working distance of $36 \mathrm{~cm}$ was $286 \mu \mathrm{W}$. S121C probe area is $70.88 \mathrm{~mm}^{2}$. The maximum irradiance was therefore $4.04 \mathrm{~W} / \mathrm{m}^{2}$. You can see that the maximum intensity of radiation emitted by the illuminator is smaller than the limit values in the current safety standards. Although exposure of eyes to a lot of LED sources may increase the intensity of radiation on the cornea, but for small distances from the eye illuminator total energy cannot be pictured in the same area of the retina, hence the different sources are not directly added as components of risk at close distance between eye and illuminator.

The measurements have established that there is no risk using the unit regardless of distance and position of the person to the device. According to the measurements shown in Fig. 3 it can be concluded that radiation illuminator in both configurations is not a threat to vision. In addition, these measurements showed that the illuminator radiation poses no threat to others which may appear in the beam illumination.

\section{Optimization}

To ensure the best possible conditions for image acquisition by the sensor it was necessary to obtain the best possible lighting conditions. In addition, an important aspect was to ensure that effects occur necessary for the correct operation of the eye tracking algorithms [5-7].

The field of view camera has dimensions approximately $15 \times 11 \mathrm{~cm}^{2}$, hence the most important aspect to offset the spectral power distribution of illumination in the area of images was taken by the camera. This was realized by setting the LED so as to obtain maximum power at a distance of about $90 \mathrm{~cm}$ from the illuminator in a central location in an image. This allowed reducing the radiation power emitted by the illuminator without a parallel decrease of brightness. The result is shown in Fig. 7 where we see the maximum radiation power at the center of the image. Due to the small area of illumination required it is possible to increase the brightness of LEDs by using a smaller beam divergence related to the threat, however, the illuminator at the maximum radiated power will exceed the safe limit. In addition, when working with a sensor it has been observed that too strong illumination of the face makes it difficult to operation algorithms by reducing the contrast between the pupil and the iris or the appearance of areas erroneously recognized as the eye.

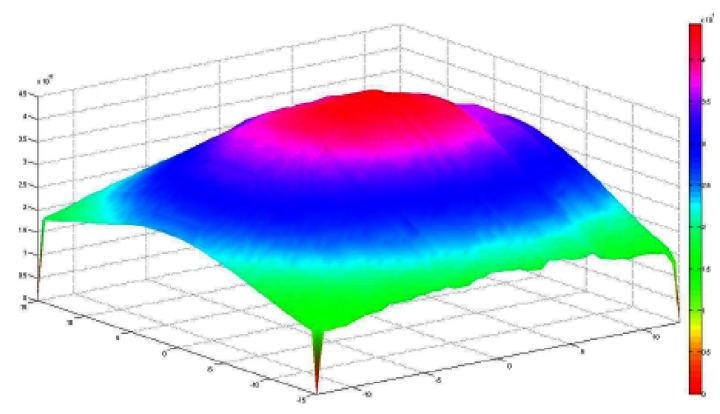

Fig. 7. The distribution of radiation power of illuminator after optimization.

An additional tool to improve the image may be the use of image processing algorithms. An example of such algorithm can be histogram equalization algorithm [8, 9], application of which can significantly increase the contrast of images obtained which reduces the power output necessary for the proper lighting the object. Example of use histogram equalization algorithm is presented in Fig. 8.

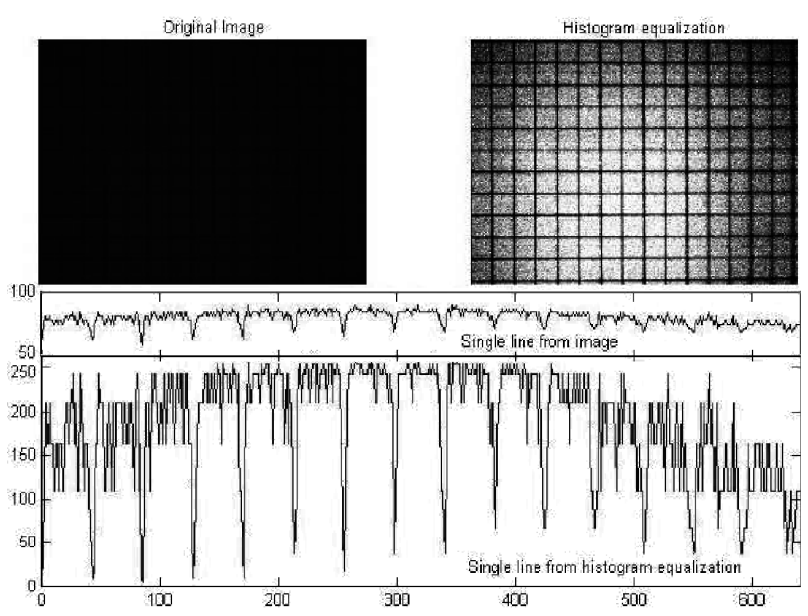

Fig. 8. Histogram equalization method used on image from camera. 
The illegible original picture (left) was machined using a histogram equalization algorithm (right) resulting in a significant improvement in contrast and distinguishing ability of the elements at the image. Under the photographs there is presented a single line of the images before and after applying the algorithm. Using more sophisticated and complex algorithms makes it possible to obtain more precise data for the algorithms responsible for keeping an eye at constant parameters of illuminator.

\section{Conclusions}

The study allowed us to confirm that the illuminator, the sensor at the maximum power level, in accordance with current standards, is not dangerous to the eyes of the person examined, or to the public. It was also found that prolonged exposure to illuminator radiation is not a threat to device user. Furthermore, the tests allowed the determination parameters of illuminator providing optimal conditions for the operation of the eye activity detection algorithms that minimize the negative effects of too low or too high a level of lighting the subject's face.

Besides, there were proposed possible ways to improve performance of the device to minimize the radiation power emitted by the illuminator, while maintaining optimal parameters for recording images by the camera of the sensor. These solutions provide unchanged parameters of the algorithms for investigating the condition of the driver, while improving the safety of user eyes.

\section{References}

[1] K. Różanowski, K. Murawski, Acta Phys. Pol. A 122, 874 (2012).

[2] C. Tyszkiewicz, T. Pustelny, Opt. Appl. 34, 507 (2004).

[3] Polish Norm PN-EN 60825-1 (in Polish).

[4] Order of the Polish Minister of Labour and Social Politics since 29.XI.2002 concerning highest permissible concentrations of agents in work places, Dz.U. 2002 No. 217, position 1833 (in Polish).

[5] K. Murawski, Methods Models Automat. Robot. 76, 356 (2010).

[6] T. Pustelny, J. Ignac-Nowicka, B. Jarzabek, A. Burian, Opt. Appl. 34, 551 (2004).

[7] K. Mikolajczyk, Int. J. Computer Vision 65, 137 (2005).

[8] R.C. Gonzalez, R.E. Woods, Digital Image Processing, 3rd ed., Wiley, London 2008.

[9] R. Garg, B. Mittal, S. Garg, Metal Inject. Mould. 2, 1397 (2011). 